Aus der chirurgischen Abteilung des herzoglichen Krankenhauses zu Braunschweig.

\title{
Zur Kasuistik und Diagnose der Torsion des Skrotalhodens.
}

\author{
Ion Dr. H. F. Brunzel.
}

(Nit 3 Abbildungen.)

\begin{abstract}
Nachdem im Jahre I $885 \mathrm{~N}$ i c o la d o n i 2 Fälle von Torsion des Samenstranges mit hämorrhagischer Infarzierung des erkrankten Hodens beschrieben hatte, hat sich die Hodentorsion als besonders eigenartige Erkrankung ständig des Interesses der Chirurgen erfreut. La uenstein konnte infolgedessen in seiner bekannten Arbeit über die Torsion des Hodens (Volkmanns Sammlung klinischer Vorträge, neue Folge Nr. 92, Chirurgie Nr. 26) bereits I 894 über I 2 Fälle dieser Art zusammenfassend berichten. I9I I war die Kasuistik schon auf 50 Fälle angewachsen, wenn wir einer Notiz von Till mann s (Lehrbuch der Chirurgie, IX. Auflage, II. Teil, Seite 540) folgen, der eine Arbeit von B o g$\mathrm{d}$ a n ik zitiert. Inzwischen dürfte die Zahl einschlägiger Beobachtungen wohl das erste Hundert erreicht haben. Da aber schätzungsweise kaum die Hälfte der Fälle von Hodentorsionen den bereits völlig deszendierten und im Scrotum liegenden Hoden betraf, wird eine weitere Kasuistik hierzu doch noch nicht überflüssig sein. Allerdings sind die grundlegenden Anschauungen über die Vorbedingungen, die erst zur Erkrankung führen können, über die rein mechanisch zu erklärende Pathogenese, über die pathologische Anatomie und die nur chirurgische Therapie schon von $L$ a ue $n$ stein und seinen Vorgängern ausreichend klargelegt, so daß Wesentliches durch kasuistische Beiträge kaum noch hinzugefügl werden kann. Nur die Diagnose der Hodentorsion, wcnige; bei den Leistenhoden als bei den bereits deszendierten Scrotalhoden, ist noch nicht so sicher festgelegt, daß nicht weitere
\end{abstract}


Beobachtungen nützen könnten. Sind doch die meisten Hodentorsionen unter falscher Diagnose operiert worden. Und gerade die rechtzeitige Diagnose und dadurch ermöglichte Frühoperation kann erst das rein theoretische Interesse an der Frage der Hodentorsion in ein dann auch praktisches umwandeln, wenn es dadurch gelingt, den torquierten Hoden als funktionierendes Organ erhalten zu können. Leider aber hat das Krankheitsbild der Hodentorsion das unverdiente Schicksal, immer gerade dann verkannt zu werden orler überhaupt unbekannt zu sein, wenn man zuerst einen Fall zu beobachten und zu behandeln Gelegenheit hat.

So haben wir in unserm ersten Fall cbenfalls die Diagnose vor der Operation nicht gestellt. Bei der retrospektiven Betrachtung des Falles, warum wir dazu nicht imstande waren, und wie in der Folge derartige Fehler zu vermeiden gewesen wären, haben wir uns an der Hand des Studiums des durch Kastration gewonnenen Hodenpräparates ein Symptom zurechtlegen können, das zweifellos bei unserem ersten Fall vorhanden gewesen sein muß, aber trotz seiner Sinnfälligkeit übersehen wurde. Da wir annehmen, daß dieses Symptom, wenn nicht überhaupt ganz unbekannt, so doch noch nicht genügend bekannt ist, hatten wir schon nach der Beobachtung des ersten Falles im Jahre I9I3 die Absicht, wenn sich seine Zuverlässigkeit bei einem neuen Fall erwies, zumal uns das Kennzeichen pathognomonisch für die Torsion des Scrotalhodens zu sein schien, dieses Symptom zu beschreiben. Wir haben unsere erste Beobachtung bereits in Mai 1913 im Ärzteverein zu Braunschweig mitgeteilt und ausdrücklich auf die Bedeutung dieses Symptoms für die Diagnose hingewiesen. Aber erst jetzt nach 4 Jahren ist uns ein zweiter Fall von Hodentorsion zur Beobachtung gekommen und da ließ das Symptom - einseitiger Hochstand und Fixierung des erkrankten Hodens durch die verkürzende Torsion des Samenstranges -- in der Tat die richtige Diagnose leicht und mit Sicherheit sofort stellen. Der Beweis für die Zuverlässigkeit des eben mitgeteilten Kennzeichens der 'Torsion des Scrotalhodens war somit erbracht.

Wir wollen genetisch verfahren und die Frage so darstellen, wie sie sich an der Hand des ersten Falles uns entwickelt hat. 
So mögc denn zunächst die Krankengeschichte des ersten Falles folgen.

F all I. R. N., 24 Jahre alt, Maler, aus B. Aufgenommen am I3., entlassen am 28. V. 1913. Der Patient war vor 5 Tagen, ohne äußere Veranlassung, mit heftigen Schmerzen in der rechten leiste erkrankt. Die Schmerzen ließen vorübergehend wohl nach, wurden aber allmählich fast unerträglich. Nach der Operation wurde noch ergänzend festgestellt, daß der Descensus beider Hoden erst im zwölften Lebensjahre erfolgte. Der rechte Hoden sei stets etwas kleiner wie der linke gewesen und so beweglich, daß der Patient ihn leicht habe umdrehen können. Auch habe er früher schon ofter bei der Arbeit ähnliche Schmerzattacken gehabt, dic aber rasch vorüber gegangen seien.

B ef und: Temperatur 37,5 Grad, Puls normal, Lungen o. B. Die rechte Scrotalhälfte ist enorm empfindlich, so daB eine genauere Palpation kaum móglich ist. Die Haut sieht entzündlich infiltriert und ödematös aus. Der rechte Hoden und Nebenhoden und ebenso der Samenstrang, der sich verdickt anfuhlt, sind ebenfalls spontan und auf leise Berïhrung schon heftig schmerzend. Bei dem verheirateten Patienten läßt sich eine Gonorrhoe ausschließen.

Die Diagnose wurde auf alte Tuberkulose mit frischem entzundlichem Nachschub gestellt.

Bei der Operation in Narkose lieB sich folgender Befund feststellen: Die auffallend weite Tunica raginalis propria enthalt in mäßiger Menge hàmorrhagisches Exsudat. Nach dessen Entfernung liegt der dunkelblau, wie eine abgeschnürte Darmschlinge verfärbte Hode zutage. Er hängt an einem stark entwickelten Mesorchium, das durch Torsion nach der medialen Seite die Gefäße des Samen. stranges abgeklemmt hat, nach der nun leicht ermöglichten Detorquierung frei in der Tunica vaginalis propria, wie „die Frucht an ihrem Stiele". Da nach dem ganzen Aussehen des Hodens und der Dauer der Torsion an eine Erholung nicht zu denken war, folgte dic Kastration des rechten Hodens. Naht der ödematösen Scrotalhaut. Verband.

Der Verlauf gestaltete sich glatt und nach der $p p$. erfolgten Heilung wurde der Patient am 28. V. 1913 beschwerdefrei aus dem Krankenhaus entlassen.

Wir haben also die richtige Diagnose des Falles vor der Operation nicht gestellt. Die lokalen entzündlichen Erscheinungen an der rechten Scrotalhälfte verführten uns zu der Annahme einer tuberkulösen Erkrankung des Nebenhodens, um so eher, als eine Gonorrhöe bei dem verheirateten Patienten sich ausschließen Jieß. 
Das durch die Kastration gewonnene Präparat wurde 1913 bei der Demonstration im Ärzteverein mit herumgezeigt. Jetzt liegt uns nur noch die damals für die Demonstration nach dem Präparat gezeichnete Abbildung vor, die die Verhältnisse nach der Hochklappung der breit eröffneten Tunica vaginalis propria rom Hoden in medialer Ansicht zeigt (vgl. Fig. I).

Man sieht, daß auch in unserem Fall die als besonders typisch fur die Torsion allgemein bekannten Vorbedingungen erfüllt sind.

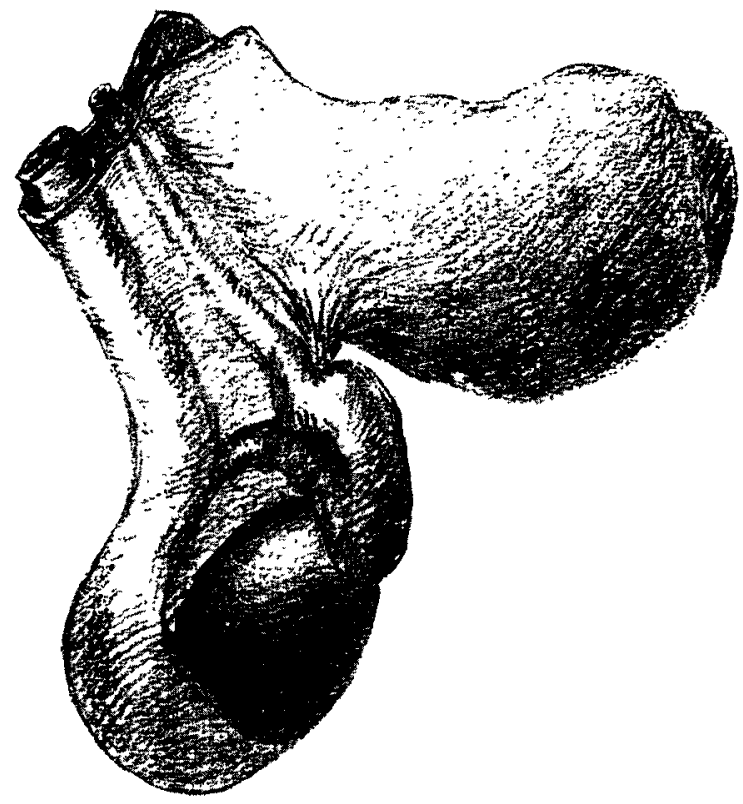

Fig. $\mathrm{r}$.

Hoden des ersten Falles. Die Tunica vag. propria ist aufgeschnitten und hoclgeklappt. Ansicht des Hodens von der medialen Seite. Die Torsion ist zurückgebracht.

Die Tunica vaginalis propria ist auffallend weit. Der Hode hängt frei in ihr an einem stark entwickelten Mesorchium. Der Ductus deferens und der Plexus pampiniformis verlaufen getrennt voneinander und bilden so ein ziemlich breites Auflängeband für den Hoden. Der Hode weist auch die von K o ch e r beschriebene Inversio testis horizontalis auf, der ebenfalls nach ihm eine be. sondere Bedeutung für das Zustandekommen der Torsion zuzu- 
sprechen ist. Als Nebenbefund ergibt sich auch ein abnormes Verhalten des Nebenhodens zum Hoden selbst. Er ist nur am Kopf- und Schwanzteil mit dem Hoden verbunden, während der Körper des Nebenhodens sich bogenförmig vom Hoden entfernt.

Auch in diesem Fall hat es sich um einen verspätet deszendierten Hoden gehandelt, der von der Torsion betroffen wurde. Nach der Angabe des Patienten seien beide Hoden erst etwa von zwölften Lebensjahre ab im Scrotum zu fühlen gewesen. Auch sei der rechte Hode kleiner als der linke gewesen und so beweglich, daß er ihn habe „umdrehen" können.

Die mikroskopische Untersuchung ergab ausgedehnte hämor. rhagische Infarzierung. Vom eigentlichen Hodengewebe waren kaum noch Spuren zu entdecken. Einen gleichen Befund bot übrigens auch der Nebenhode. Makroskopisch sah der infarzierte Hode auf dem Durchschnitt wie ein praller Bluterguß in einen abgeschlossenen Hohlraum aus. Nach allem war an eine Jrholung des Hodenparenchyms also nicht zu denken. Die Kastration ist zweifellos zu Recht erfolgt.

Wesentlich sollen uns an diesem Fall aber nur diagnostische Gesichtspunkte interessieren.

Spannte man das durch die Operation gewonnene Präparat derart an, daß die eine Hand den gesamten Samenstrang an der Abtragungsstelle fixierte, während die andere Hand den Hoden nach der Detorsion festhielt und rief nun die bei der Operation gefundene Torsion des Hodens nach der medialen Seite hervor, und zwar um I80 Grad, so fiel sofort die Annäherung der beiden fixierenden Hände dabei auf. Der Samenstrang erfuhr also cine wesentliche Verkürzung. Wir erinnerten uns nun auch mit einem Male deutlich, daß der torquierte rechte Hode auffällig nahe dem äußeren Leistenring bei der Operation aufgefunden wurde, also hoch fixiert war. Erst nach ausgiebiger Spaltung der Tunica, an deren oberem Ende der Hode gelegen hatte, und der nun leicht möglichen Detorquierung näherte der Hode sich dem unteren Pol derselben und erreichte damit auch einen symmetrischen Stand zum linken Hoden. Es mußte ferner sofort einleuchten, daß mit zunehmender Breite des durch den Samenstrang gebildeten Mesorchiums bei gleicher Torsion eine höhere Aufdrehung des Hodens erfolgen mußte oder daß schon eine geringere Torsion cinen 
gleichen Hochstand des Hodens bedingte. Immer aber mußte jede Hodentorsion für den Scrotalhoden einen höheren Stand ergc'sen als ihn der nicht torquierte hatte, und der Unterschied in der Stellung beider Hoden mußte stets feststellbar sein. Wir glaubten durch diese einfachen Überlegungen, die man sich auch leicht aus der beigefügten Zeichnung ableiten kann (vgl. Fig. I), in der den Samenstrang mehr oder weniger verkürzenden Torsion und in dem dadurch bedingten einseitigen Hochstand des erkrankten Hodens ein pathognomonisches Zeichen für die 'Torsion des Scrotalhodens gefunden zu haben.

Es ist uns nicht bekannt, daß dies Symptom schon beschrieben worden ist. Aber selbst wenn dies der Fall wäre, dürfte seine praktische Bestätigung im zweiten von uns beobachteten Fall und die dadurch mit Sicherheit ermöglichte Diagnose den nochmaligen Hinweis darauf nicht überflüssig erscheinen lassen. Damit kommen wir zur Mitteilung des zweiten Falles.

Fall 2. A. MI, 18 Jahre alt, Postaushelfer, aus $F$ Aufgenom. men am I7. IV., entlassen am I. V. I9I7.

Der Patient war vor neun Tagen erkrankt mit plötzlich eintretenden Schmerzen im linken Hoden. Eine Veranlassung dafür vermochte der Patient nicht anzugeben. Er wurde vom Arzt draußen mit Kalkwasserumschlägen behandelt und schließlich als Nebenhodenentzündung zur Aufnahme ins Krankenhaus geschickt. Gonorrhoe bestand nicht. Auf besonderes Befragen gab der Patient noch an, daß ihm von einem verspateten Descensus der Hoden nichts bekannt sei. Die Hoden hätten sich schon stets im Scrotum befunden. Er habe aber schon dreimal gleiche Anfalle von Schmerzen im linken Hoden in den letzten Jahren gehabt, nur seien sie schon nach kurzer Zeit von selbst besser geworden, und er habe deswegen die Arbeit niemals ausgesetzt.

Der Befund war folgender: Die linke Scrotalhälfte ist ödematös und dic Haut deutlich gerotet. Der linke Hoden erschcint diffus geschwollen und ist lebhaft empfindlich. Er steht deutlich höher als der rechte an normaler Stelle befindliche Hoden (siehe Fig. 2). Der Hode läßt sich nicht zu gleichem Stand mit dem rechten herab. ziehen, obwohl vorher nach der präzisen Angabe des Patienten beide Hoden gleich tief gestanden hätten. Es fand sich also in ausgeprägter Weise ein einseitiger fixierter Hochstand des erkrankten linken Hodens. Die Temperatur betrug 36,8 Grad.

Damit stand fur uns die Diagnose: Hodentorsions chon vor der Operation fest. 
Freilegung des Hodens. Dieser ist zweimal um I 80 Grad in der ziem'ich weiten Tunica vaginalis propria nach rechts gedreht. Die Tunica enthält wenig hämorrhagische Flüssigkeit und ist leicht mit Fibrinniederschlägen bedeckt, die an der Rückseite des Hodens lockere Verklebungen mit ihm eingegangen sind. Der Hode ist dunkelblauschwarz verfärbt, und behält diese Färbung auch nach der leicht möglichen Rückdrehung bei. Bei dem langen Bestande der Erkrankung ist an eine Erholung des Hodenparenchyms nicht zı denken. Kastration. Naht der ödcmatösen Haut des Scrotum, die leicht bräunlich verfärbt ist durch die Diffusion von Blutbarbstoff.

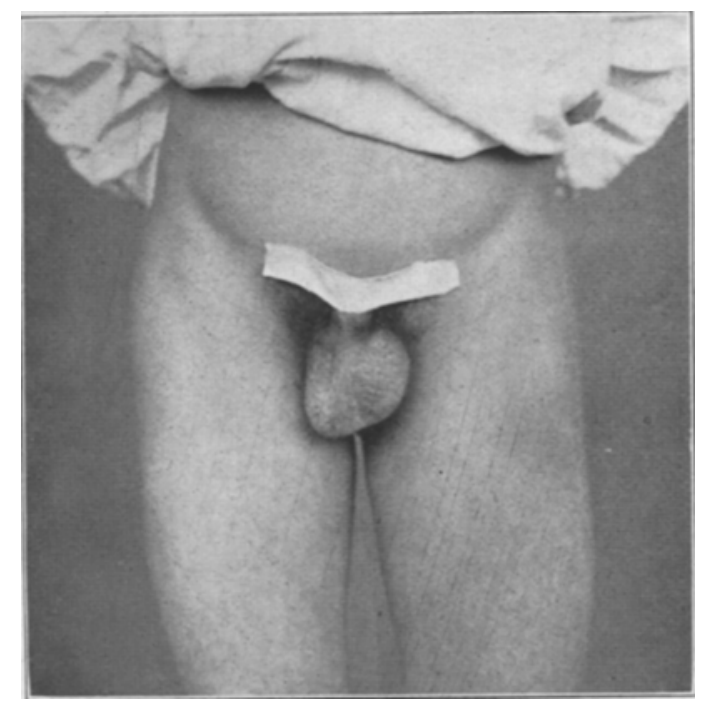

Fig. 2.

Photographie der Scrotalgegend des zweiter Falles vor der Operation. Man crkennt deutlich den Hochstand des linken Hodens und die Anschwellung der linken Scrotalhalfte.

Der Verlauf gestaltet sich glatt. Der Patient wird am I. V. rgr 7 geheilt und beschwerdefrei entlassen.

Die Fig. 3 gibt den Hoden in der torquierten Stellung naturgetreu wieder. An dem frischen Präparat ließ sich wieder ein langes Mesorchium feststellen. Der Hode zeigte eine mäßig ausgesprochene Inversio testis horizontalis; Ductus deferens und Plexus pampiniformis verliefen aber nicht getrennt. Ebenso war der Nebenhoden in ganzer Ausdehnung dem Hoden anliegend. 
Die Tunica vaginalis war ganz erheblich weiter als normal, aber geringer entwickelt als beim ersten Falle.

Wieder wäre bei der Diagnose irreführend gewesen die Rötung und das Ödem der Scrotalhaut, die leicht zur Annahme hätten führen können, daß es sich um eine entzündliche Affektion handele. Bei dem Ausschluß von Gonorrhöe lag wieder der Gedanke an Tuberkulose nahe. Der einseitige Hochstand und die Fixierung des erkrankten Hodens der sich vor der Erkrankung nach der bestimmten Angabe des Patienten an völlig analoger

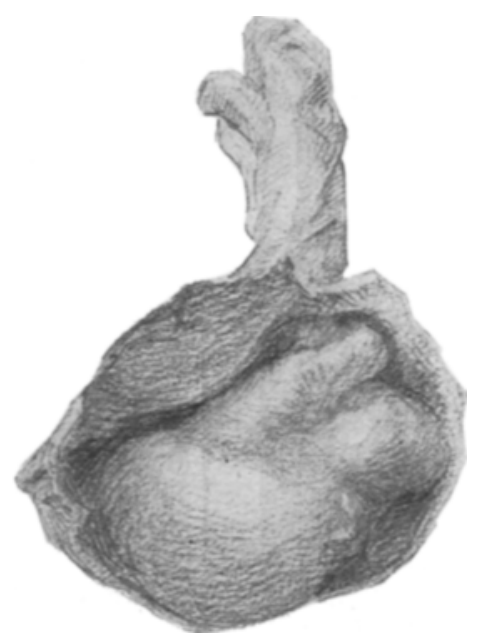

Iig. 3 .

Hoden des zweiten Falles. Tunica vag. propria eroffnet. Ansicht von vorn. Die Torsion besteht noch.

Stelle wie der gesunde rechte Hode befunden haben sollte, führte ohne weiteres zu der sicheren Diagnose, daß eine Torsion des Scrotalhodens vorliege. Die Diagnose wird in den ersten Stunden nach der Torsion sozar noch leichter zu stellen sein, da dann noch dic sekundären entzündlichen Erscheinungen an der Scrotalhaut fehlen werden.

Leider konnte in diesem Falle bei dem neuntägigen Bestand der Torsion die sofortige Operation nichts mehr zur Erhaltung des Hodens beitragen. Wenn auch für den Leistenhoden eine 
frühzeitige Operation bei der häufigen Minderwertigkeit und Neigung zur bösartiger Degeneration derselben, wenn man ihn nicht verlagern kann, meist wenig nützt, so ist für den Scrotalhoden die früheste Frühoperation zur Erhaltung eines funktionstüchtigen Organs durchaus anzustreben.

Wir wissen ja aus den bekannten Untersuchungen $\mathrm{M}$ if $\mathrm{let} \mathrm{s}$ (Archiv für klin. Chir. Bd. 24, I 879) wie außerordentlich empfindlich das Parenchym des Hodens gegen Störungen der lokalen Blutzirkulation ist. M if let sagt an dritter Stelle der Ergebnisse seiner ,Untersuchungen über die pathologischen Veränderungen des Hodens, welche durch Störungen der lokalen Blutzirkulation veranlaßt werden“, folgendes: „Das Drüsengewebe des Hodens ist gegen jede Störung der Blutzirkulation in den Gefäßen des Samenstranges außerordentlich empfindlich; nicht nur bei gleichzeitiger Unterbrechung der Blutzufuhr seitens der Arteria spermatica interna und Behinderung des Abflusses durch die Venen des Samenstranges, sondern auch, wenn letztere Zirkulationsstörungen einzeln für sich auftreten. Stets zerfällt das Drüsengewebe rasch, so daß ein Schwund des Hodens die Folge ist. Dieser vollzieht sich unter mehr oder minder starker Bindegewebswucherung." Als weitere Stütze für diese Ausführungen Miflets möge hier auch noch auf das Ergebnis der Versuche Enderlens hingewiesen sein (Klinische und experimentelle Studien zur Frage der Torsion des Hodens. Deutsche Zeitschr. für Chir. Bd. 43, I896, Seite I86). En de r len fand, daß eine Unterbindung der Samenstranggefäße nur bis zur 16 stündigen Dauer keinen schädlichen Einfluß ausübte. „Erst als der Seidenfaden 22 Stunden liegen blieb, stellte sich deutlich Größenabnahme der operierten Seite neben derberer Konsistenz ein. Nach zwei Monaten wog der intakte Testikel I I g, derjenige der operierten Seite 2,75 g. Auf der Schnittfläche des letzteren fanden sich derbe Bindegewebszüge, welche kleine Cysten umschlossen. Spermatozoen fehlten. Mikroskopisch fand sich starke Erweiterung der Hodenkanälchen, Abflachung, Abstoßung des Epithels, bedeutende Zunahme des Bindegewebes."

Daß die Behandlung der Hodentorsion also moglichst früh einzusetzen hat, ist klar; und ebenso, daß sie rein chirurgisch sein sollte. 
Der Eingriff hätte, wenn der Hoden noch erhalten werden kann, dann nach erfolgter Rückdrehung nur in der Beseitigung. der erweiterten Tunica vaginalis propria $z u$ bestehen, nach $W$ in $\mathrm{kelmann}$ oder wohl besser noch nach $v$. Bergmann, und in der Befestigung des Hodens. Eventuell dürfte die Schaffung von fixierenden Verklebungen des Hodens mit der Scrotalwand nach Jodierung der Wunde unter leichter Tamponade hierbei von Nutzen sein, um eine neuerliche Torsion des Hodens mit möglichster Sicherheit dauernd auszuschließen. Auch e in e Revision der anatomischen Verhältnisse des gesunden Hodens könnte sich gelegentlich empfehlen. Jedenfalls sollte man den Patienten nach der Operation der einen Seite stets auf die Gefahr und Möglichkeit des Eintrittes einer Torsion auf der bisher gesunden Seite aufmerksam machen und dem Patienten dringend nahe legen, wenn die ihm bereits bekannten Erscheinungen auf der bisher gesunden Scite auftreten, sich unverzüg. lich zur operativen Behandlung einzufinden.

Den Hoden einfach unblutig zurück zudrehen, wie es z. B. Anders Prag möglich war (Zentralblatt für Chir. 1915, Nr. I9. Seite 343), sollte wenig Nachahmung finden, da ein Rückfall olne Beseitigung der die Torsion ermöglichenden anatomischen Vorbedingungen um so eher zu befürchten ist, als verschiedentlich von den Autoren berichtet worden ist, daß der torquierte Samenstrang auch noch nach der Detorsion eine deutliche Neigung zur Einnahme seiner pathologischen Drehungsstellung zeigte. Uibrigens hatte auch der Patient von Prag nach seinen Angaben schon früher einmal eine solche Torsion durchgemacht.

La uenstein hatte zum SchluB seiner bereits erwähnten Arbeit auf eine von $K$ üstner hervorgehobene und anderen bestätigte Beobachtung hingewiesen, daß rechtsseitige Ovarialtumoren eine linksspiralige, linksseitige eine rechtsspiralige Stieldrehung zu erfahren pflegen. Er hatte aus der Tatsache, daß in 5 Fällen von rechtsseitigem Leistenhoden eine linksspiralige Drehung des Samenstranges bestand, für die Torsion der Scrotalhoden ein analoges Verhalten angenommen. Unsere beiden Fälle können als Bestätigung dafür bei ihrer jedesmal nach der medialen Seite erfolgten Torsion gelten, da sie also im selben Sinne wie die Ovarialtumoren torquiert waren. 
Selbst wenn das von uns an der Hand des durch die Kastration des ersten Falles gewonnenen Präparates abgeleitete Symptom des einseitigen fixierten Hodenhochstandes als pathognomonisches Kennzeichen der Torsion des $\mathrm{Scrotalhodens}$ bereits beschrieben sein sollte, so dürfte der nochmalige Hinweis darauf in der vorliegenden Arbeit nicht nutzlos sein. Die Diagnose der Hodentorsion wird zweifellos dadurch erleichtert und gesichert. Und bei allgemein verbreiteter Kenntnis des beschriebenen Symptoms dürfte auch die Hodentorsion für die Frühoperation zur Erhaltung eines funktionstüchtigen Organs mehr als bisher zugänglich gemacht werden können. Als Beitrag dazu ist diese Arbeit hauptsächlich gedacht. 